\title{
Erratum
}

\section{Biquandles of small size and some invariants of virtual and welded knots}

[J. Knot Theory Ramifications, Vol. 20, No. 7 (2011), pp. 934-954; DOI: $10.1142 / \mathrm{S} 0218216511009042]$

\author{
Roger Andrew Fenn* and Andrew Bartholomew ${ }^{\dagger}$ \\ School of Mathematical Sciences, \\ University of Sussex, Falmer, \\ Brighton, BN1 9RH, UK \\ *rogerf@sussex.ac.uk \\ †andrewb@layer8.co.uk
}

Received 7 September 2016

Accepted 11 April 2017

Published 1 June 2017

\begin{abstract}
Some of the results on welded knots in the title paper were incorrect. In this corrigendum, these are corrected and extended. An additional appendix is also included.

Keywords: Biquandles; generalized knots.

Mathematics Subject Classification 2010: 16B99, 17A99, 57M27
\end{abstract}

\section{Introduction}

Analysis of the results presented in the first version of this paper, [1, revealed an ambiguity in the utilization of the theory. This led to an example of a welded knot which was claimed to be nontrivial, but was in fact trivial. Moreover, this ambiguity resulted in incorrect examples being used in some of the proofs and in the appendix presenting the computer output.

The ambiguity was a confusion on the ordering of the elements of the braid which meant that the wrong forbidden move was used. Here, we resolve the ambiguity and correct the examples both in the text and in the appendix. We also add a second appendix giving some interesting examples of welded knots.

\footnotetext{
${ }^{*}$ Corresponding author.
} 


\section{Ordering Braid Strands, etc.}

The ambiguity in the first version relates to the ordering of braid strands. So, it is important that we get these details correct from the start. We consider braids to be projected onto the page so that their strands are oriented horizontally from left to right, reflecting the order in which we write braid words. With this view, we order braid strands from the bottom upwards. For example, the braid below is written as $\sigma_{2} \sigma_{1} \tau_{3} \sigma_{4}^{-1}$.

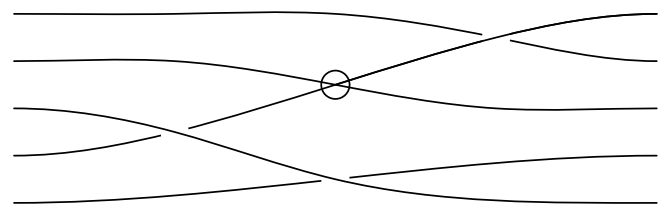

The symbol $\sigma^{ \pm}$stands for a classical crossing, either positive or negative. The symbol $\tau$ stands for a virtual or weld crossing. Since $\tau^{2}=1$, it follows that $\tau^{-1}=\tau$.

\subsection{Diagram symmetries}

In order to make sure that no further confusion occurs, we will look at the symmetries of virtual and welded diagrams.

Reflection in the Plane In this symmetry, the underlying 4-valent graph is unchanged, but the positive classical crossings are turned into negative ones and vice versa. The virtual or weld crossings remain the same.

Let $K$ represent a braid or knot diagram. The result of this symmetry is written $\bar{K}$ and is called the mirror image of $K$. Geometrically, it corresponds to a reflection in the plane of the diagram. For the braid example above is $\bar{K}=\sigma_{2}^{-1} \sigma_{1}^{-1} \tau_{3} \sigma_{4}$.

Inversion or Reflection in a Vertical Line For a braid, this reverses the order of the generators and their signs. It is written $-K$ and is called the reverse of $K$. So, in our example, the reverse braid is $\sigma_{4} \tau_{3} \sigma_{1}^{-1} \sigma_{2}^{-1}$. For a knot diagram, the inverse is obtained by reversing its orientation and the signs of its crossings. In the classical case, the inverse of a knot is sometimes defined by reversing its orientation, but not the signs of its crossings. This would be represented by the combination $-\bar{K}$.

The classical knot $K=9_{32}$ has all symmetric versions $K, \bar{K},-K$ and $-\bar{K}$ distinct.

Reflection in a Horizontal Line Choose a horizontal line south of the diagram $K$ and reflect the underlying 4-valent graph. Give the corresponding classical crossings the opposite sign, the virtual crossings remain the same. Let $K^{*}$ denote the result of the reflection in a horizontal line. For our example, braid $K^{*}$ is the braid $\sigma_{3}^{-1} \sigma_{4}^{-1} \tau_{2} \sigma_{1}$.

The flip Choose a horizontal line south of the diagram $K$ and reflect the underlying 4-valent graph. Give the corresponding crossings the same sign. The result is written 
$K_{f}$ and is called the flip of $K$. Note that $K_{f}=\overline{K^{*}}=\bar{K}^{*}$. Under a flip, our braid is converted into $\sigma_{3} \sigma_{4} \tau_{2} \sigma_{1}^{-1}$. Geometrically, this corresponds to a three-dimensional rotation about the line. For a classical knot, this will not change its type. However, this is not the case for a virtual or welded knot as we will see.

We have been unable to find an example of a welded knot in which all eight symmetries are distinct.

\subsection{Weld conditions}

In the theory of welded knots, the first forbidden move, $F 1$, shown below is allowed.

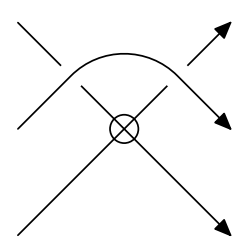

$F 1$

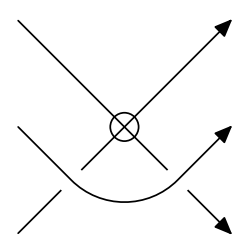

Regarded as braids on three strands and following the bottom-up convention on braid strand ordering this move assert the equivalence of $\sigma_{2}{ }^{-1} \tau_{1} \sigma_{2}$ and $\sigma_{1} \tau_{2} \sigma_{1}{ }^{-1}$.

If $S$ and $T$ are a pair of switches used to calculate a welded or virtual braid invariant, then they must respect the fact that the above move is or is not allowed.

We write $S_{i}$ for the operation of the switch $S$ between the $i$ th and $(i+1)$ th strands of a braid, so that $S_{i}=I^{n-i-1} \times S \times I^{i-1}$. Thus, for the braid describing the first forbidden move above $S_{1}=I \times S$ and $S_{2}=S \times I, S_{1}(a, b, c)=(a, S(b, c))$, $S_{2}(a, b, c)=(S(a, b), c)$, and so on. We may therefore express the condition imposed on switches $S$ and $T$ by the first forbidden move as $S_{2}^{-1} T_{1} S_{2}=S_{1} T_{2} S_{1}^{-1}$; that is $\mathbf{F}_{\mathbf{1}} \cdot T_{1} S_{2} S_{1}=S_{2} S_{1} T_{2}$.

In the theory of welded knots, the second forbidden move, $F 2$ shown below, is not allowed.
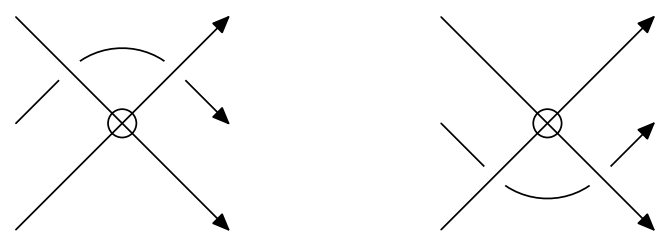

$F 2$

We express the condition imposed on $S$ and $T$ by the second forbidden move as $S_{2} T_{1} S_{2}^{-1}=S_{1}^{-1} T_{2} S_{1}$; that is

$\mathbf{F}_{\mathbf{2}} . S_{1} S_{2} T_{1}=T_{2} S_{1} S_{2}$. 
The convention used for writing $S_{i}$ and $T_{i}$ in the relations $F_{1}$ and $F_{2}$ and the bottom-up strand numbering used to describe the geometry of a braid were not explicitly stated in the first version of the paper.

\section{The significance of braid strand ordering}

The ordering of braid strands is significant in the case of welded knots whereas it is not in the case of classical knots. If the reverse convention of numbering braid strands top-down were adopted, the allowed first forbidden move expressed in braid form as $\sigma_{2}{ }^{-1} \tau_{1} \sigma_{2}=\sigma_{1} \tau_{2} \sigma_{1}{ }^{-1}$ would become
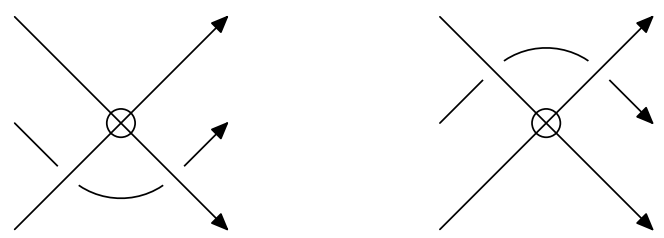

which is, in fact, the disallowed second forbidden move. Reversing the braid strand ordering is equivalent to the flip symmetry $K_{f}=\bar{K}^{*}$ and the reflection in the plane, $\bar{K}$, interchanges the allowed and disallowed weld conditions.

It is worth noting that a similar situation exists with virtual knot theory, where we consider knots in thickened surfaces of some genus. Here also, reversing the braid strand ordering is a combination of a reflection in the surface and in a horizontal line and not all virtual braid closures are invariant under this action.

In summary, we may restate the relevant part of Sec. 4 of the first version using the above conventions.

\section{Virtual and Weld Invariants}

A biquandle is said to have order $k$ if $k$ is the smallest positive integer for which $S^{k}$ is the identity. A pair of biquandles $(S, T)$ is a virtual invariant if

V. $T$ has order two and $T_{1} S_{2} T_{1}=T_{2} S_{1} T_{2}$.

A virtual invariant pair is called an essential virtual pair if neither of the forbidden conditions

$\mathbf{F}_{\mathbf{1}} \cdot T_{1} S_{2} S_{1}=S_{2} S_{1} T_{2}$,

$\mathbf{F}_{\mathbf{2}} \cdot S_{1} S_{2} T_{1}=T_{2} S_{1} S_{2}$

are satisfied.

A virtual invariant pair is said to be an essential welded pair if the pair satisfies the first forbidden move $F_{1}$, but do NOT satisfy the second forbidden move $F_{2}$.

Theorem 5.1. In Theorem 5.1 of the original paper, the example given is not consistent with the above definition of an essential virtual pair. The correct example is

$$
\begin{aligned}
& S: B Q_{3}^{4}, \quad U=(\iota, \iota,(243),(234)), \quad D=(\iota,(34),(34),(34)), \\
& T: B Q_{30}^{4}, \quad U=((34),(34),(34),(34)), \quad D=((34),(34),(34),(34)) .
\end{aligned}
$$


Thus, $S$ and $T$ form an essential virtual pair.

Let $\psi_{1}=\sigma_{3}^{-1} \sigma_{2} \sigma_{1}^{2} \sigma_{2} \sigma_{4}^{3} \sigma_{3} \sigma_{2}, \psi_{2}=\sigma_{4}^{-1} \sigma_{3} \sigma_{2} \sigma_{1}^{-2} \sigma_{2} \sigma_{1}^{2} \sigma_{2}^{2} \sigma_{1} \sigma_{4}^{5}, \phi_{1}=\sigma_{4} \sigma_{5}^{-1} \sigma_{2}^{-1} \sigma_{1}$ and $\phi_{2}=\sigma_{4}^{-1} \sigma_{5}^{2} \sigma_{2} \sigma_{1}^{-2}$. In [2], Bigelow defines the braids $b_{1} \in B_{5}$ and $b_{2} \in B_{6}$ as

$$
b_{1}=\left[\psi_{1}^{-1} \sigma_{4} \psi_{1}, \psi_{2}^{-1} \sigma_{4} \sigma_{3} \sigma_{2} \sigma_{1}^{2} \sigma_{2} \sigma_{3} \sigma_{4} \psi_{2}\right] \quad \text { and } \quad b_{2}=\left[\phi_{1}^{-1} \sigma_{3} \phi_{1}, \phi_{2}^{-1} \sigma_{3} \phi_{2}\right] \text {. }
$$

The number of fixed points of $f_{(S, T)}$ corresponding to $b_{1}$ is 736 , whereas the number of fixed points of the unlink with five components $4^{5}=1024$. The number of fixed points of $f_{(S, T)}$ corresponding to $b_{2}$ is 1648 , whereas the number of fixed points of the unlink with six components is $4^{6}=4096$.

Theorem 5.2. Similarly, the examples in Theorem 5.2 are inconsistent with the above definition of an essential virtual pair. With the notation above and putting $b=b_{2} \tau_{1} \sigma_{2} \tau_{3} \tau_{4} \tau_{5} \tau_{6}$, the correct examples are

$$
\begin{aligned}
& S: B Q_{6}^{3}, \quad U=((12),(23),(13)), \quad D=((123),(123),(123)), \\
& T: B Q_{7}^{3}, \quad U=((123),(123),(123)), \quad D=((132),(132),(132)),
\end{aligned}
$$

which is an essential virtual pair for which the number of fixed points of $f_{(S, T)}$ corresponding to $K=b b b$ is 9 , and

$$
\begin{aligned}
& S: Q_{3}^{3}, \quad U=((23),(13),(12)), \quad D=\iota, \\
& T: Q_{1}^{3}, \quad U=\iota, \quad D=\iota,
\end{aligned}
$$

which is an essential welded pair for which the number of fixed points of $f_{(S, T)}$ corresponding to $K=b b b$ is 9 .

The essential virtual pair,

$$
\begin{aligned}
S: B Q_{53}^{4}, & U=((1234),(1432),(1234),(1432)), \\
D & =((1234),(1432),(1234),(1432)), \\
T: B Q_{26}^{4}, & U=(\iota, \iota,(12)(34),(12)(34)), \quad D=(\iota, \iota,(12)(34),(12)(34)),
\end{aligned}
$$

given in the proof of Theorem 5.2 shows that $K_{3}$ is nontrivial as a virtual knot is consistent with the above definition of an essential virtual pair and does not require updating.

\section{Appendix A. The Computer Output}

We reproduce Secs. A.1 and A.2 in their entirety. For a complete list of results and diagrams, see www.layer8.co.uk/maths/biquandles. 


\section{A.1. Welded knots}

Having calculated lists of biquandles, it is trivial to determine the essential welded pairs of biquandles. There are two such pairs of size 3

$$
\begin{aligned}
\text { P1. } & S: B Q_{3}^{3}, \quad U=(\iota,(132),(123)), \quad D=((23),(23),(23)), \\
& T: B Q_{5}^{3}, \quad U=((23),(23),(23)), \quad D=((23),(23),(23)), \\
\text { P2. } & S: Q_{3}^{3}, \quad U=((23),(13),(12)), \quad D=\iota, \\
& T: Q_{1}^{3}, \quad U=\iota, \quad D=\iota
\end{aligned}
$$

and 8 of size 4 :

$$
\begin{aligned}
& \text { P3. } S: B Q_{3}^{4}, U=(\iota, \iota,(243),(234)), \quad D=(\iota,(34),(34),(34)), \\
& T: B Q_{9}^{4}, \quad U=(\iota,(34),(34),(34)), \quad D=(\iota,(34),(34),(34)), \\
& \text { P4. } S: B Q_{14}^{4}, \quad U=(\iota,(34),(143),(134)), \quad D=((34),(34),(34),(34)), \\
& T: B Q_{30}^{4}, \quad U=((34),(34),(34),(34)), \quad D=((34),(34),(34),(34)), \\
& \text { P5. } S: B Q_{19}^{4}, \quad U=(\iota,(13)(24),(14)(23),(12)(34)), \\
& \quad D=((243),(243),(243),(243)), \\
& T: B Q_{39}^{4}, \quad U=((234),(234),(234),(234)), \\
& D=((243),(243),(243),(243)),
\end{aligned}
$$

P6. $S: B Q_{29}^{4}, \quad U=(\iota,(34), \iota, \iota), \quad D=((34),(34),(14),(13))$, $T: B Q_{30}^{4}, \quad U=((34),(34),(34),(34)), \quad D=((34),(34),(34),(34))$,

P7. $S: B Q_{37}^{4}, \quad U=((234),(23),(34),(24)), \quad D=((243),(234),(234),(234))$,

$$
T: B Q_{40}^{4}, \quad U=((234),(243),(243),(243)),
$$

$$
D=((243),(234),(234),(234))
$$

P8. $S: B Q_{54}^{4}, \quad U=((12)(34),(12)(34),(12)(34),(12)(34))$,

$$
D=((1342),(1243),(1243),(1342)),
$$

$T: Q_{1}^{4}, \quad U=\iota, \quad D=\iota$,

P9. $S: Q_{3}^{4}, \quad U=((243),(134),(142),(123)), \quad D=\iota$, $T: Q_{1}^{4}, \quad U=\iota, \quad D=\iota$,

P10. $S: Q_{7}^{4}, \quad U=((24),(14), \iota,(12)), \quad D=\iota$, $T: Q_{1}^{4}, \quad U=\iota, \quad D=\iota$. 
The website also lists the seven essential welded pairs of quandle-related biquandles of size five and the 40 essential pairs of quandle-related biquandles of size six. We include a selection of the essential welded pairs of size five and six here for reference below.

$$
\begin{aligned}
\text { P12. } & S: Q_{1}^{5}, \quad U=((2345),(1354),(1425),(1532),(1243)), \quad D=\iota, \\
& T: Q_{21}^{5}, \quad U=\iota, \quad D=\iota, \\
\text { P13. } & S: Q_{2}^{5}, \quad U=((23)(45),(14)(35),(15)(24),(13)(25),(12)(34)), \quad D=\iota, \\
& T: Q_{21}^{5}, \quad U=\iota, \quad D=\iota, \\
\text { P43. } & S: Q_{4}^{6}, \quad U=((2453),(1364),(1562),(1265),(1463),(2354)), \quad D=\iota, \\
& T: Q_{72}^{6}, \quad U=\iota, \quad D=\iota, \\
P 46 . & S: Q_{10}^{6}, \quad U=((2453),(1543),(1425),(1352),(1234), \iota), \quad D=\iota, \\
& T: Q_{72}^{6}, \quad U=\iota, \quad D=\iota .
\end{aligned}
$$

Given that the fixed point invariant $F_{n}$ takes the value $n$ for the unknot, it is possible to use the list of essential welded pairs to search for distinct nontrivial welded knots. Using this technique, the following knots have been distinguished.

\begin{tabular}{|c|c|}
\hline Knot & Braid word \\
\hline w3.1 & $\sigma 1 \tau 2 \sigma 3-\sigma 2-\sigma 2-\sigma 1 \tau 2-\sigma 3 \sigma 2$ \\
w3.2 & $\tau 1-\sigma 2 \tau 1-\sigma 1-\sigma 1 \tau 2$ \\
w4.2 & $-\sigma 1-\sigma 2 \sigma 3 \tau 2 \sigma 1-\sigma 4 \sigma 3 \tau 2 \sigma 3 \sigma 4-\sigma 3-\sigma 2$ \\
w4.3 & $-\sigma 4-\sigma 3 \sigma 2 \tau 3 \sigma 4-\sigma 1 \sigma 2 \tau 3 \sigma 2 \sigma 1-\sigma 2-\sigma 3$ \\
w4.4 & $-\sigma 1 \sigma 2 \sigma 3 \tau 2 \sigma 1-\sigma 4 \sigma 3 \tau 2 \sigma 3 \sigma 4-\sigma 3 \sigma 2$ \\
w4.5 & $-\sigma 4 \sigma 3 \sigma 2 \tau 3 \sigma 4-\sigma 1 \sigma 2 \tau 3 \sigma 2 \sigma 1-\sigma 2 \sigma 3$ \\
w4.6 & $-\sigma 1 \sigma 2 \sigma 3 \tau 2 \sigma 1-\sigma 4 \sigma 3-\sigma 2 \sigma 3 \sigma 4-\sigma 3 \tau 2$ \\
w4.7 & $\tau 2 \sigma 1-\sigma 2 \tau 2 \sigma 2 \sigma 1$ \\
w4.8 & $-\sigma 1 \sigma 2-\sigma 3 \tau 2 \tau 1-\sigma 2 \tau 2 \sigma 3 \sigma 2$ \\
w4.9 & $\sigma 1-\sigma 2 \sigma 3 \sigma 2 \tau 2-\sigma 1-\sigma 2 \tau 2 \sigma 2-\sigma 3 \tau 2$ \\
w5.1 & $\sigma 3-\sigma 2 \sigma 1 \tau 2 \sigma 2-\sigma 3 \sigma 2 \tau 2-\sigma 2-\sigma 1 \tau 2$ \\
w5.2 & $\sigma 3 \tau 2 \sigma 3 \sigma 1 \tau 2 \sigma 1-\sigma 2$ \\
w6.1 & $-\sigma 2 \tau 2-\sigma 1 \tau 1 \sigma 1-\sigma 2 \tau 1-\sigma 1$ \\
w6.2 & $-\sigma 1 \sigma 2 \tau 1-\sigma 3-\sigma 2 \sigma 1-\sigma 2 \sigma 3 \sigma 2$ \\
w6.3 & $\sigma 1-\sigma 2 \tau 1-\sigma 3 \sigma 2-\sigma 1 \sigma 2 \sigma 3-\sigma 2$ \\
w6.4 & $\tau 1-\sigma 2-\sigma 2-\sigma 1-\sigma 1 \tau 2-\sigma 1-\sigma 2$ \\
w6.5 & $-\sigma 1 \tau 3-\sigma 2 \sigma 3 \sigma 4 \tau 3 \sigma 2 \sigma 1-\sigma 5 \sigma 4-\sigma 3-\sigma 3-\sigma 2 \tau 3 \sigma 4 \sigma 5-\sigma 4 \sigma 3 \sigma 2$ \\
w6.6 & $-\sigma 5 \tau 3-\sigma 4 \sigma 3 \sigma 2 \tau 3 \sigma 4 \sigma 5-\sigma 1 \sigma 2-\sigma 3-\sigma 3-\sigma 4 \tau 3 \sigma 2 \sigma 1-\sigma 2 \sigma 3 \sigma 4$ \\
w6.7 & $-\sigma 1 \tau 3-\sigma 2 \sigma 3 \sigma 4 \tau 3 \sigma 2 \sigma 1-\sigma 5 \sigma 4-\sigma 3-\sigma 3-\sigma 2-\sigma 3 \sigma 4 \sigma 5-\sigma 4 \tau 3 \sigma 2$ \\
\hline
\end{tabular}




\begin{tabular}{|c|c|}
\hline Knot & Braid word \\
\hline w6.8 & $-\sigma 5 \tau 3-\sigma 4 \sigma 3 \sigma 2 \tau 3 \sigma 4 \sigma 5-\sigma 1 \sigma 2-\sigma 3-\sigma 3-\sigma 4-\sigma 3 \sigma 2 \sigma 1-\sigma 2 \tau 3 \sigma 4$ \\
w6.9 & $-\sigma 1 \tau 3-\sigma 2-\sigma 3 \sigma 4 \tau 3 \sigma 2 \sigma 1-\sigma 5-\sigma 4 \sigma 3 \sigma 3-\sigma 2 \tau 3-\sigma 4 \sigma 5-\sigma 4-\sigma 3 \sigma 2$ \\
w6.10 & $-\sigma 5 \tau 3-\sigma 4 \tau 3 \sigma 2-\sigma 3 \sigma 4 \sigma 5-\sigma 1 \sigma 2-\sigma 3-\sigma 3-\sigma 4-\sigma 3 \sigma 2 \sigma 1-\sigma 2 \tau 3 \sigma 4$ \\
w6.11 & $\tau 1 \sigma 2-\sigma 3-\sigma 2-\sigma 2 \tau 2-\sigma 1 \tau 2-\sigma 2 \sigma 3 \sigma 2$ \\
w6.12 & $\tau 3 \sigma 2-\sigma 1-\sigma 2-\sigma 2 \tau 2-\sigma 3 \tau 2-\sigma 2 \sigma 1 \sigma 2$ \\
w6.13 & $\tau 1 \sigma 2-\sigma 3 \sigma 2 \sigma 2 \tau 2 \sigma 1 \tau 2 \sigma 2 \sigma 3 \sigma 2$ \\
w6.14 & $\tau 3 \sigma 2-\sigma 1 \sigma 2 \sigma 2 \tau 2 \sigma 3 \tau 2 \sigma 2 \sigma 1 \sigma 2$ \\
w6.15 & $\tau 1-\sigma 2-\sigma 3 \sigma 2 \sigma 2 \tau 2 \sigma 1 \tau 2 \sigma 2 \sigma 3-\sigma 2$ \\
w6.16 & $\tau 3-\sigma 2-\sigma 1 \sigma 2 \sigma 2 \tau 2 \sigma 3 \tau 2 \sigma 2 \sigma 1-\sigma 2$ \\
w6.17 & $-\sigma 1-\sigma 2 \sigma 3 \tau 2 \sigma 4-\sigma 3 \tau 2 \sigma 1-\sigma 5-\sigma 4 \sigma 3-\sigma 2 \sigma 3 \sigma 4 \tau 3 \sigma 5-\sigma 4-\sigma 3-\sigma 2$ \\
w6.18 & $\sigma 3-\sigma 2 \sigma 1-\sigma 2 \tau 2 \tau 3 \tau 1-\sigma 1-\sigma 2$ \\
w6.19 & $-\sigma 1 \sigma 2-\sigma 3 \sigma 2 \tau 2 \tau 1 \tau 3 \sigma 3 \sigma 2$ \\
w6.20 & $-\sigma 1-\sigma 3 \tau 2-\sigma 1-\sigma 3 \tau 2-\sigma 1-\sigma 3 \tau 2$ \\
w7.1 & $-\sigma 1 \sigma 2 \sigma 3-\sigma 2 \sigma 4 \tau 3 \tau 2 \sigma 1-\sigma 5-\sigma 4-\sigma 3 \sigma 2$ \\
w7.2 & $-\sigma 3 \sigma 4-\sigma 3 \sigma 5-\sigma 4-\sigma 3 \sigma 2$ \\
w7.3 & $-\sigma 3 \sigma 2-\sigma 1-\sigma 2-\sigma 2 \tau 3-\sigma 2-\sigma 3 \tau 2 \sigma 1 \sigma 2$ \\
w7.4 & $\tau 1 \sigma 2 \sigma 3 \tau 2 \sigma 1-\sigma 2 \sigma 1 \sigma 3 \sigma 2$ \\
w8.1 & $-\sigma 3 \sigma 2 \tau 3-\sigma 1 \sigma 2-\sigma 3-\sigma 4$ \\
\hline
\end{tabular}

The following table shows the fixed point invariants for the above knots determined by those essential welded pairs required to distinguish them as distinct nontrivial welded knots.

\begin{tabular}{|c|c|c|c|c|c|c|}
\hline \multirow{2}{*}{ Knot } & \multicolumn{6}{|c|}{ Size of $F_{n}$ for pair } \\
\cline { 2 - 7 } & P1 & P5 & P12 & P13 & P43 & P46 \\
\hline w3.1 & 9 & 4 & 5 & 5 & 6 & 6 \\
w3.2 & 9 & 16 & 5 & 5 & 30 & 6 \\
w4.1 & 9 & 4 & 5 & 5 & 6 & 26 \\
w4.2 & 9 & 4 & 25 & 5 & 6 & 6 \\
w4.3 & 3 & 4 & 25 & 25 & 6 & 6 \\
w4.4 & 3 & 4 & 5 & 25 & 6 & 26 \\
w4.5 & 3 & 4 & 5 & 25 & 6 & 6 \\
w4.6 & 3 & 16 & 5 & 5 & 6 & 6 \\
w4.7 & 3 & 16 & 5 & 25 & 6 & 6 \\
w4.8 & 3 & 4 & 25 & 5 & 6 & 6 \\
w4.9 & 3 & 4 & 5 & 5 & 6 & 26 \\
w5.1 & 9 & 4 & 5 & 5 & 30 & 6 \\
w5.2 & 9 & 16 & 5 & 5 & 6 & 6 \\
w6.1 & 9 & 4 & 25 & 5 & 30 & 6 \\
\hline
\end{tabular}




\begin{tabular}{|c|c|c|c|c|c|c|}
\hline & \multicolumn{5}{|c|}{ Size of $F_{n}$ for pair } \\
\cline { 2 - 7 } Knot & P1 & P5 & P12 & P13 & P43 & P46 \\
\hline w6.2 & 9 & 4 & 5 & 5 & 30 & 26 \\
w6.3 & 27 & 64 & 5 & 5 & 150 & 6 \\
w6.4 & 9 & 16 & 5 & 5 & 54 & 6 \\
w6.5 & 9 & 4 & 5 & 25 & 6 & 6 \\
w6.6 & 9 & 4 & 5 & 25 & 6 & 26 \\
w6.7 & 9 & 16 & 5 & 25 & 30 & 6 \\
w6.8 & 9 & 4 & 5 & 25 & 30 & 6 \\
w6.9 & 9 & 4 & 25 & 25 & 6 & 6 \\
w6.10 & 9 & 16 & 5 & 25 & 6 & 6 \\
w6.11 & 9 & 16 & 5 & 5 & 6 & 26 \\
w6.12 & 9 & 16 & 25 & 5 & 30 & 6 \\
w6.13 & 9 & 16 & 25 & 25 & 30 & 6 \\
w6.14 & 9 & 16 & 5 & 25 & 30 & 26 \\
w6.15 & 9 & 16 & 5 & 5 & 30 & 26 \\
w6.16 & 9 & 16 & 25 & 5 & 6 & 6 \\
w6.17 & 9 & 4 & 25 & 5 & 30 & 26 \\
w6.18 & 3 & 16 & 5 & 5 & 6 & 26 \\
w6.19 & 3 & 16 & 25 & 5 & 6 & 6 \\
w6.20 & 27 & 64 & 5 & 5 & 126 & 6 \\
w7.1 & 3 & 4 & 25 & 5 & 6 & 26 \\
w7.2 & 9 & 64 & 5 & 25 & 30 & 6 \\
w7.3 & 9 & 4 & 5 & 5 & 54 & 6 \\
w7.4 & 27 & 16 & 5 & 5 & 126 & 6 \\
w8.1 & 9 & 16 & 5 & 25 & 54 & 6 \\
\hline
\end{tabular}

In the original paper, we stated that we had discovered a nontrivial welded knot with trivial fundamental quandle and so trivial fundamental group [3]. This would have answered a question in [5. However, this was incorrect and the question remains open.

\section{A.2. Birack series}

The series of coefficients described in Sec. 6 can be used to distinguish classical and virtual knots from the unknot. We append here a table of coefficients which generate the repeating invariants for the unknot, the trefoil, Figs. 2 and 8 of the Kishino knots, see [4]. The virtual pairs $(S, T)$ were constructed with $S$ chosen to be a birack, shown in the table using the above numbering scheme, and $T$ set to the twist in each case. 


\begin{tabular}{|c|c|c|c|c|}
\hline \multirow[b]{2}{*}{ Knot } & \multirow[b]{2}{*}{ Braid word } & \multicolumn{3}{|c|}{ Coefficient series for virtual pair constructed from } \\
\hline & & $B R_{130}^{6}$ & $B R_{261}^{6}$ & $B R_{3878}^{6}$ \\
\hline & unknot & 633 & 64 & 6 \\
\hline 3.1 & $\sigma_{1} \sigma_{1} \sigma_{1}$ & 1299 & 1618 & 18 \\
\hline 4.1 & $\sigma_{1} \sigma_{2}^{-1} \sigma_{1} \sigma_{2}^{-1}$ & 633 & 1816 & 6 \\
\hline K1 & $\sigma_{1} \sigma_{2}^{-1} \sigma_{1}^{-1} \tau_{2} \sigma_{1} \sigma_{2} \sigma_{1}^{-1} \tau_{2}$ & 633 & 64 & 12 \\
\hline $\mathrm{K} 2$ & $\sigma_{1}^{-1} \sigma_{2}^{-1} \sigma_{1} \tau_{2} \sigma_{1}^{-1} \sigma_{2} \sigma_{1} \tau_{2}$ & 1299 & 64 & 12 \\
\hline
\end{tabular}

\section{Appendix B.}

\section{B.1. Braid ordering}

The welded knot that is the closure of $\tau_{2} \sigma_{1} \sigma_{2}^{-1} \tau_{2} \sigma_{2} \sigma_{1}$ is shown by the fixed point invariants in the table in Sec. A.1 to be a nontrivial welded knot (w4.6). However, if this braid was drawn using the alternative convention of numbering braid strands top down, rather than using the adopted convention of bottom-up numbering, then the resultant diagram would actually be the braid $\tau_{1} \sigma_{2} \sigma_{1}^{-1} \tau_{1} \sigma_{1} \sigma_{2}$ (based on the adopted convention). Seiichi Kamada has shown explicitly that the closure of this braid is equivalent to the unknot.

An example of the variance of virtual braids under the flip symmetry, $K_{f}$, is given by the essential virtual pair

$$
\begin{aligned}
& S: B Q_{6}^{3}, \quad U=((12),(23),(13)), \quad D=((123),(123),(123)), \\
& T: B Q_{7}^{3}, \quad U=((123),(123),(123)), \quad D=((132),(132),(132)) .
\end{aligned}
$$

The number of fixed points $f_{(S, T)}$ corresponding to the braid $b_{3}=$ $\sigma_{1} \sigma_{2}^{-1} \sigma_{3} \sigma_{2}^{-1} \tau_{2} \sigma_{1}^{-1} \sigma_{2} \sigma_{3}^{-1} \tau_{2}$ is zero, whereas the number of fixed points $f_{(S, T)}$ corresponding to the flip of this braid, $b_{3 f}=\sigma_{3} \sigma_{2}^{-1} \sigma_{1} \sigma_{2}^{-1} \tau_{2} \sigma_{3}^{-1} \sigma_{2} \sigma_{1}^{-1} \tau_{2}$, is three. For reference, $K, K_{f}$, the closures of $b_{3}$ and $b_{3 f}$ are shown in the following diagram:
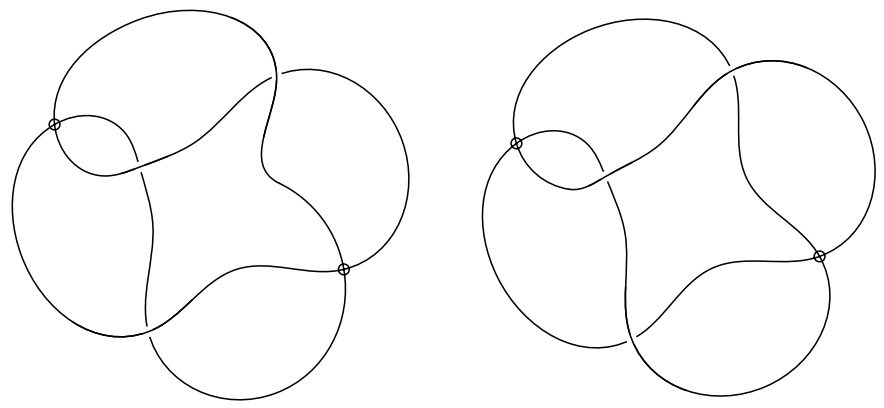

Two distinct virtual knots $K$ and $K_{f}$ 


\section{B.2. A welded knot distinct from its reverse}

As can be seen from the table in Sec. A.1, using the essential welded pair P12, the number of fixed points for the welded braid

$$
w 4.1=\sigma_{1}^{-1} \sigma_{2} \sigma_{3} \tau_{2} \sigma_{1} \sigma_{4}^{-1} \sigma_{3} \tau_{2} \sigma_{3} \sigma_{4} \sigma_{3}^{-1} \sigma_{2}
$$

is 25. The number of fixed points using $P 12$ for the flip of $w 4.1$,

$$
w 4.2=\sigma_{4}^{-1} \sigma_{3} \sigma_{2} \tau_{3} \sigma_{4} \sigma_{1}^{-1} \sigma_{2} \tau_{3} \sigma_{2} \sigma_{1} \sigma_{2}^{-1} \sigma_{3}
$$

is 5 . Thus, $P 12$ distinguishes between the closure of these two welded braids.

The closures of these braids correspond to the same welded knot diagram, but with the reversed orientation.

It is also the case that $w 4.3$ and $w 4.4$ represent a pair of distinct welded knots that are each other's orientation reverse.

\section{References}

[1] A. Bartholomew and R. Fenn, Biquandles of small size and some invariants of virtual and welded knots, J. Knot Theory Ramifications 20(7) (2011) 943-954, arXiv:1004.1320v1.

[2] S. Bigelow, The Burau representation is not faithful for $n=5$, Geom. Topol. 3 (1999) 397-404.

[3] R. Fenn, A strange torus embedding, preprint.

[4] R. Fenn, M. Jordan and L. Kauffman, Biquandles and virtual links, Topology Appl. 145 (2004) $157-175$.

[5] R. Fenn, L. Kauffman and V. Manturov, Virtual knot theory-unsolved problems, Fund. Math. 188 (2005) 293-323. 